\title{
Review of: "Reproductive skew, fitness costs, and winner-loser effects in social-dominance evolution"
}

\section{Maria Aparecida Visconti ${ }^{1}$}

1 Universidade de São Paulo

Potential competing interests: The author(s) declared that no potential competing interests exist.

The article is very good. The authors apply the game theory to analise the reproductive sucess related to social rank. The text is clear as well as the figures and table. 